\title{
Global Cerebral Blood Flow Increase Reveals Focal Hypoperfusion in Schizophrenia
}

\author{
Stephan F. Taylor, M.D., Rajiv Tandon, M.D., and Robert A. Koeppe, Ph.D.
}

Recent functional neuroimaging strategies have evaluated cerebral blood flow (CBF) to determine specific sites of action of pharmacologic agents. Since many pharmacologic agents change global CBF, we investigated the effects of global $C B F$ changes on regional perfusion with acetazolamide, which increases global CBF via nonneuronal mechanisms. We used the $\left[{ }^{15} \mathrm{O}\right] P E T$ technique to measure CBF before and after we infused 8 schizophrenic patients and 10 healthy control subjects with acetazolamide. The rostral anterior cingulate cortex demonstrated a greater perfusion increase in the schizophrenic subjects after acetazolamide infusion, relative to other areas of the brain. During the baseline condition, this area showed relative hypoperfusion in our sample of schizophrenic subjects, consistent with previous functional neuroimaging studies. The results demonstrate the need for caution in interpreting CBF changes after pharmacologic challenge, because global $C B F$ changes can confound the assessment of regionallyspecific pharmacologic action.

[Neuropsychopharmacology 21:368-371, 1999]

(C) 1999 American College of Neuropsychopharmacology.

Published by Elsevier Science Inc.
KEY WORDS: Schizophrenia; Cerebrovascular circulation; Emission computed tomography; Acetazolamide; Gyrus cinguli

A recent application of functional neuroimaging techniques to the study of schizophrenia involves the measurement of cerebral activity under pharmacological challenge, e.g., using apomorphine (Dolan et al. 1995) or ketamine (Lahti et al. 1995). While perturbations of regional activity may demonstrate how neurotransmitter systems exert regionally-specific effects on brain function, interpretation of results is confounded by several factors, such as polysynaptic effects, non-specific changes and the interference of pharmacologic doses of drug on the coupling between the

From the Departments of Psychiatry (SFT, RT) and Internal Medicine Division of Nuclear Medicine (RAK), University of Michigan, and Department of Psychiatry (SFT) Veterans Administration Medical Center, Ann Arbor, Michigan.

This work was previously presented at the Second International Conference on Functional Mapping of the Human Brain, Boston, MA, in June, 1996.

Address correspondence to: Stephan F. Taylor, M.D., Department of Psychiatry, University of Michigan Medical Center, UH 9D Box 0118, 1500 E. Medical Center Drive, Ann Arbor, MI 48109-0118

Received June 23, 1998; accepted October 5, 1998. neuroimaging signal and neuronal activity. Another important confound occurs in global cerebral blood flow (CBF) changes, which are usually not measured in activation paradigms relying on relative activity changes or the fMRI BOLD signal. In fact, agents such as apomorphine or ketamine do increase global cerebral blood flow (Edvinsson et al. 1993), but the effect of global CBF changes on relative perfusion in schizophrenia is not well-understood.

In order to investigate the hemodynamic response of the cerebral vasculature in schizophrenia, we infused subjects with acetazolamide, which reversibly inhibits carbonic anhydrase, decreasing intravascular $\mathrm{pH}$ and thereby increases CBF throughout the brain (Vorstrup et al. 1984; Bonte et al. 1988). Our data demonstrate that merely by increasing global flow, areas hypoperfused in the baseline state show regionally-enhanced increase in a sample of schizophrenic patients.

\section{MATERIALS AND METHODS}

We recruited 8 stable schizophrenic patients (3 inpatients prior to discharge and 5 outpatients; 3 females, 5 
males), whose diagnoses were established by structured clinical interview according to DSM-IV criteria (American Psychiatric Association 1994). All were taking psychotropic medications (6 on clozapine). Mean age $( \pm \mathrm{SD})$ was $38.0 \pm 5.8$ years, and mean duration of illness was $15.3 \pm 5.7$ years. Ten ( 4 females, 6 males), psychiatrically-healthy comparison subjects were selected to match the age range of the patient subjects (mean $=35.8$ years, $\mathrm{SD}=6.2$ ). After complete description of the study to all subjects, written informed consent was obtained.

PET scans were performed with a Siemens CTI 931/ 08-12 camera (CTI Inc., Knoxville, TN). For each emission scan, subjects received an i.v. bolus injection of 66 $\mathrm{mCi}$ of $\left[{ }^{15} \mathrm{O}\right] \mathrm{H}_{2} \mathrm{O}$. In 7 controls and 5 patients, arterial samples provided data for an input function and quantitative calculation of CBF. Subjects underwent a total of 6 PET scans (60 sec acquisition); we focus here only on a baseline scan, which was pseudo-randomized with the first 5 scans and the 6th scan, obtained fifteen minutes after an i.v. infusion of $1 \mathrm{gm}$ of acetazolamide. During these two scans, subjects had their eyes covered, were instructed to lie still and heard only ambient noise from cooling fans. Acetazolamide infusion always occurred prior to the last scan. The other scans involved presentation of visual stimuli and are reported elsewhere (Taylor et al. 1997).

An automated algorithm (Minoshima et al. 1992, 1993, 1994) transformed each reconstructed image to a standardized atlas system (Talairach and Tournoux 1988). Count data were proportionally normalized to the mean activity for gray-matter voxels (arbitrarily set to 1000), and the images were averaged within each group. Images were smoothed to an effective full width at half-maximum of $\sim 13 \mathrm{~mm}$. The mean difference for each normalized voxel during the acetazolamide condition compared to the baseline condition was expressed as a Z-score, and corrected probabilities for rejecting the null hypothesis of no difference were calculated (Worsley et al. 1992). By examining differences in the normalized pattern of (relative) CBF, we searched for activity in the schizophrenic subjects which either increased more or less than the rest of the brain after acetazolamide. The center-of-mass atlas coordinates of significant foci enabled the placement of a spherical volume of interest (13.5 $\mathrm{mm}$ diameter) on the anatomically-standardized image of each subject, permitting comparison between groups.

\section{RESULTS}

No subjects experienced any subjective effects of acetazolamide during the PET scan, although a few noted mild parasthesias within 2 minutes of injection. With acetazolamide, both groups showed the expected, global increase in CBF $(39.2 \pm 20.7[\% \pm \mathrm{SD}$ ] for normals and $54.1 \pm 38.1$ for schizophrenics, $\mathrm{t}=-0.79$, NS). An analysis of variance revealed no group effects on global $\mathrm{CBF}$ and no interaction between scan and group. Examination of the difference image in the schizophrenic group (normalized acetazolamide flow minus normalized baseline flow) revealed one, well-focused positive peak which mapped to the rostral anterior cingulate cortex (ACC; coordinates: $3 \mathrm{~mm}$ left, $39 \mathrm{~mm}$ anterior and $18 \mathrm{~mm}$ superior of the anterior commissure; $\mathrm{Z}=$

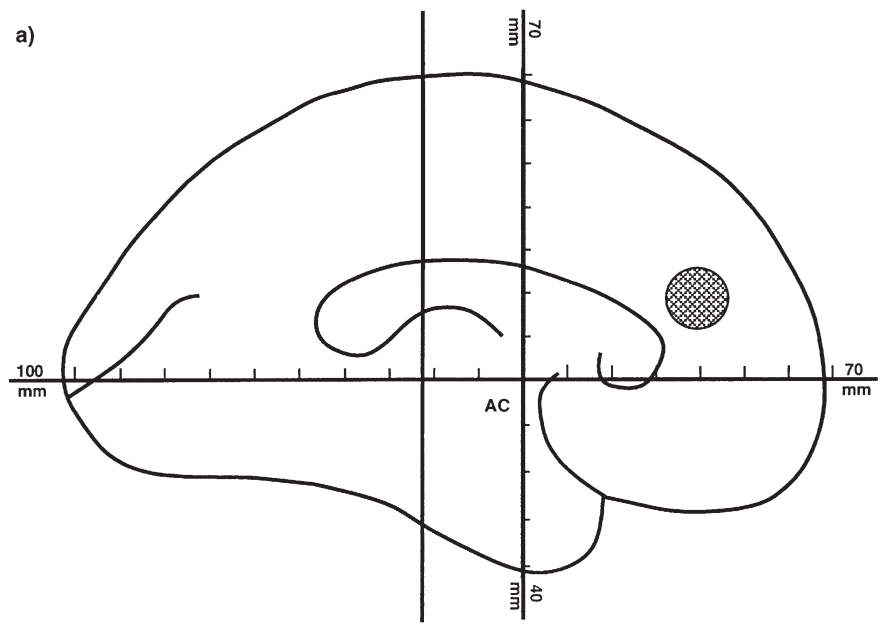

b)

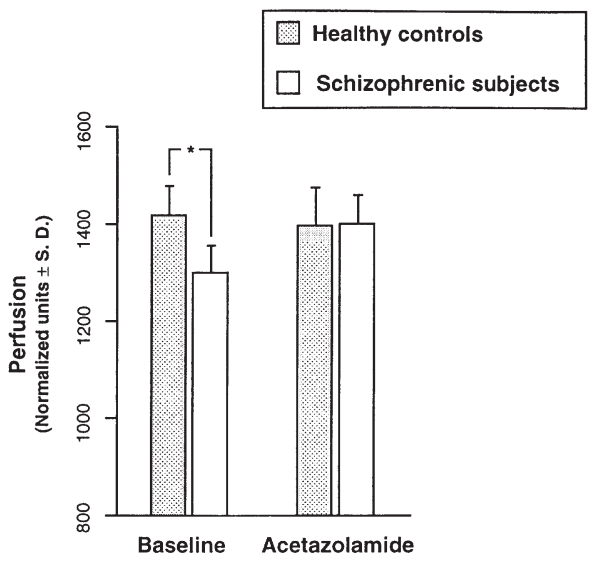

Figure 1. (a) The hatched circle in the anterior cingulate cortex shows the one area of significantly greater perfusion increase in the schizophrenic subjects after acetazolamide infusion, relative to the other areas of the brain. The diameter of the circle $(13.5 \mathrm{~mm})$ indicates the maximum cross-section of the spherical volume of interest used to analyze individual, anatomically-standardized images. (b) The graph shows that at the location indicated in (a), normalized flow was significantly less in the schizophrenic, compared to the normal, subjects during the baseline condition $\left({ }^{*} \mathrm{t}=4.13, \mathrm{df}=16, \mathrm{p}<.001\right)$. 
$4.4, p=.05$, corrected). There were no other foci, positive or negative, with $Z>3.8$. At the ACC focus, we found that whereas $\mathrm{CBF}$ during the acetazolamide condition was equal in both groups, $\mathrm{CBF}$ in the ACC was significantly less than the normal controls in this region during the baseline state (Figure 1). There were no differences between relative activity in the ACC for the two groups during acetazolamide infusion or between the infused and non-infused conditions for the healthy control group.

\section{DISCUSSION}

In our schizophrenic group, the hypoperfused ACC increased more than other regions as global CBF increased with acetazolamide. By perturbing global CBF, we identified a region of apparent "reserve flow" in the schizophrenic brain. Hypoperfusion of the ACC in unmedicated patients is consistent with findings from other groups, measuring both metabolism (Haznedar et al. 1997; Tamminga et al. 1992) and cerebral blood flow (Andreasen et al. 1992; Lewis et al. 1992). Furthermore, some groups have reported that antipsychotic medications may depress activity in the ACC/medial prefrontal cortex (Holcomb et al. 1996), a finding of possible relevance for our sample of medicated schizophrenic subjects. Whatever the etiology of reduced ACC activity in the patient group, in the comparison between the baseline scan and the acetazolamide infusion scan for the schizophrenic subjects, the ACC appeared as an "activation," probably reflecting CBF in the ACC region catching up with the normal pattern of regional CBF.

The perfusion change in the ACC suggests an important interpretation. The finding that blood flow in a hypoactive region could increase to normal levels supports the idea that reductions in metabolism or blood flow are not secondary to atrophic changes or other structural deficits. We would not conclude that by restoring flow to the ACC, we had restored function to this region; rather, we prefer the interpretation that neuronal function itself is abnormal in this region, and the metabolic demand for $\mathrm{CBF}$ is lower in the patients.

Several caveats should be kept in mind. An effect of scan order may have differentially influenced activity in the ACC, since the acetazolamide scan always followed the baseline scan in our subjects. While possible, it would be very unusual to find a region, such as the ACC, which does not activate in schizophrenia (Andreasen et al. 1992; Lewis et al. 1992), show greater activation than healthy subjects. Nevertheless, a group with a placebo infusion would be helpful to definitively establish an effect of acetazolamide on regional CBF changes. Our patients were medicated at the time of the study, which may have influenced our findings, although the effects of acetazolamide occur via alteration of intravascular $\mathrm{pH}$ and not via a neurogenic mechanism (Vorstrup et al. 1984).

These results demonstrate that global CBF changes are not uniform and may lead to erroneous interpretation of normalized, non-quantitative perfusion images. For example, Figure 1 could be interpreted as an interaction between the ACC and a pharmacologic agent, but if that agent also increased global CBF, the pharmacologic interpretation would be confounded by global CBF changes. Measurement of brain metabolism, e.g., with a $\left[{ }^{18} \mathrm{~F}\right]$ fluorodeoxyglucose PET study, may avoid this confound, since metabolism exhibits significantly less variability than CBF. While we only examined a sample of schizophrenic patients, the results suggest the need for cautious interpretation of pharmacologically-induced CBF changes in any patient group known to have regional perfusion abnormalities.

\section{ACKNOWLEDGMENTS}

This work was supported by the Ann Arbor Veterans Administration Medical Center and a grant from the Scottish Rite Schizophrenia Research Program to the first author. Subject recruitment, screening and data collection were provided by Laura Decker, Lisa Becks, and JoAnn Goodson. Thanks are due to Kirk Frey for helpful suggestions on study design.

\section{REFERENCES}

American Psychiatric Association (1994): Diagnostic and Statistical Manual of Mental Disorders, 4th ed (DSM-IV). Washington, D.C., American Psychiatric Association

Andreasen NC, Rezai K, Alliger R, Swayze VW, Flaum M, Kirchner P, Cohen G, O'Leary DS (1992): Hypofrontality in neuroleptic-naive patients and in patients with chronic schizophrenia. Arch Gen Psychiatry 49:943-958

Bonte FJ, Devous MD, Reisch JS (1988): The effect of acetazolamide on regional cerebral blood flow in normal human subjects as measured by single-photon emission computed tomography. Invest Radiol 23:564-568

Dolan RJ, Fletcher P, Frith CD, Friston KJ, Frackowiak RS, Grasby PM (1995): Dopaminergic modulation of impaired cognitive activation in the anterior cingulate cortex in schizophrenia. Nature 378:180-182

Edvinsson L, MacKenzie ET, McCulloch J (1993): Cerebral Blood Flow and Metabolism. New York, Raven Press

Haznedar MM, Buchsbaum MS, Luu C, Hazlett EA, Siegel BJ, Lohr J, Wu J, Haier RJ, Bunney WJ (1997): Decreased anterior cingulate gyrus metabolic rate in schizophrenia. Am J Psychiatry 154:682-684

Holcomb HH, Cascella NG, Thaker GK, Medoff DR, Dannals RF, Tamminga CA (1996): Functional sites of neuroleptic drug action in the human brain: PET/FDG studies with and without haloperidol. Am J Psychiatry 153:41-49

Lahti AC, Holcomb HH, Medoff DR, Tamminga CA (1995): Ketamine activates psychosis and alters limbic blood flow in schizophrenia. Neuroreport 6:869-872 
Lewis SW, Ford RA, Syed GM, Reveley AM, Toone BK (1992): A controlled study of 99m-Tc-HMPAO singlephoton emission imaging in chronic schizophrenia. Psychol Med 22:27-35

Minoshima S, Berger KL, Lee KS, A MM (1992): An automated method for rotational correction and centering of three-dimensional functional brain images. J Nuc Med 33:1579-1585

Minoshima S, Koeppe RA, Frey KA, Kuhl DE (1994): Anatomic standardization: Linear scaling and nonlinear warping of functional brain images. J Nucl Med 35:1528-1537

Minoshima S, Koeppe RA, Mintun MA, Berger K, Taylor SF, Frey KA, Kuhl DE (1993): Automated detection of the intercommissural (AC-PC) line for stereotactic localization of functional brain images. J Nuc Med 34:322-329

Talairach J, Tournoux P (1988): A Co-planar Stereotaxic Atlas of a Human Brain. Stuttgart, Thieme-Verlag
Tamminga CA, Thaker GK, Buchanan R, Kirkpatrick B, Alphs LD, Chase TN, Carpenter WT (1992): Positron emission tomographic analysis of central D1 and D2 dopamine receptor occupancy in patients treated with classical neuroleptics and clozapine. Relation to extrapyramidal side effects. Arch Gen Psychiatry 49:538-544

Taylor SF, Tandon R, Koeppe RA (1997): PET study of greater visual activation in schizophrenia. Am J Psychiatry 154:1296-1298

Vorstrup S, Henriksen L, Paulson OB (1984): Effect of acetazolamide on cerebral blood flow and cerebral metabolic rate for oxygen. J Clin Invest 74:1634-1639

Worsley KJ, Evans AC, Marrett S, Neelin P (1992): A threedimensional statistical analysis for CBF activation studies in human brain. J Cereb Blood Flow Metab 12:900-918 